\title{
Physical Exercise in the Prevention and Rehabilitation of Cardiac Patients: The Cuban Experience in the Primary Health Care System
}

\section{Eduardo Rivas-Estany*}

Department of Cardiac Rehabilitation, Institute of Cardiology and Cardiovascular Surgery, Havana, Cuba

\section{Summary}

Heart disease, particularly ischemic heart disease is the leading cause of death in Cuba, also showing a high prevalence. The benefits of physical exercise in primary and secondary prevention of this disease have been pointed out, also the favorable effects of physical training in relation to quality of life, morbidity and mortality of patients with cardiovascular diseases. To design an exercise regimen it is necessary to take into account the "fundamental principles of physical training": type of exercise, intensity, duration, frequency and individualization.

The main objective of a program of exercise is to increase the quality and quantity of life of patients, allowing them to return to community life as normally as possible, active and productive. To test the effectiveness of this program, periodic assessments can be made by the appropriate health care level specialists. One of the main benefits expected from the implementation of this exercise program is that a vast majority of patients may be reinstated earlier and in better condition to their work or social activities. It is also expected an improvement in functional capacity, symptom relief, reduction in the consumption of prescribed drugs, decreased anxiety and the usually observed depression in these patients. It will be observed, an important preservation of the role of the patient in their family and social life, all of which will produce significant socio-economic benefits.

Keywords: Exercise; Physical training; Ischemic heart disease; Myocardial infarction; Cardiac rehabilitation

\section{Introduction}

Cardiovascular diseases, considering the heart and cerebro-vascular illness, still represent the leading cause of death in Cuba for more than 50 years, with a mortality rate in 2015 of $300.9 \times 100000$ ( 218.3 and 82.6 $\times 100000$ inhabitants for heart disease and stroke, respectively) [1]. Ischemic heart disease and acute myocardial infarction have a major role in this mortality, also showing a high prevalence and incidence, respectively. This fact is more alarming when we consider that the heart attack every time appears in earlier ages of life, precisely at a time when the individual is more useful to society [2].

Several publications have demonstrated the marked benefits of physical exercise in primary and secondary prevention of ischemic heart disease, and have also pointed out the beneficial effects of physical training as part of a cardiac rehabilitation program in patients with this pathology and other cardiovascular diseases [3-6]. The prescription of physical exercises has been defined as "the recommendation of a regime of systematic and individualized physical activity to achieve optimal patient physiological benefits of exercise training [5-9]. This method will try to increase their physical capacity, improve their health and reduce the risk of recurrence of the disease and ensure their safety during participation in the exercises [10].

The specific objectives to participate in a physical training program vary according to the particular interests of the individuals, their needs, their health or their underlying disease, which influence the exercise prescription. Its indications are listed below.

\section{Indications of Physical Training}

Recent or old myocardial infarction

Chronic stable angina

Coronary Artery Bypass Surgery

Non-tributary coronary disease surgical treatment

Other surgical heart diseases (congenital or acquired)

Coronary angioplasty
Stable heart failure

Cardiomyopathy

High Blood Pressure

Heart and cardiopulmonary transplants

Pacemakers and implantable cardioverter-defibrillators

Non-lethal cardiac arrhythmias

Peripheral vascular disease

Primary or secondary prevention of ischemic heart disease

Long periods of physical inactivity

To achieve the beneficial effects of physical exercise as a primary and secondary prevention in ischemic heart disease, it is necessary that the "fundamental principles of physical training" are met, therefore to design or indicate a regimen of physical exercise the following aspects must be taken into account: type of exercises and how to perform them, intensity, duration and frequency. Also to be considered are individualization and mode of progression for those exercises [11].

The exercise program should be well suited to the type of patient and time of evolution after acute coronary event or cardiac surgery or therapeutic procedure used, either interventionist or only with medications. It is generally desirable to adapt the phase of rehabilitation in which the patient is: hospital, convalescent or maintenance.

*Corresponding author: Eduardo Rivas-Estany, Department of Cardiac Rehabilitation, Institute of Cardiology and Cardiovascular Surgery, Havana, Cuba, Tel: 537838 6140; Fax: 537834 4435; E-mail: erivas@infomed.sld.cu

Received August 05, 2016; Accepted October 26, 2016; Published November 02, 2016

Citation: Rivas-Estany E (2016) Physical Exercise in the Prevention and Rehabilitation of Cardiac Patients: The Cuban Experience in the Primary Health Care System. Primary Health Care 6: 243. doi: 10.4172/2167-1079.1000243

Copyright: ( 2016 Rivas-Estany E. This is an open-access article distributed under the terms of the Creative Commons Attribution License, which permits unrestricted use, distribution, and reproduction in any medium, provided the original author and source are credited. 


\section{Objectives}

The main objectives of an exercise program consist of the increasing and prolongation of the quality and quantity of life of cardiac patients, allowing them to function at the highest work level possible, by their own efforts, to return to life in the community as normally as possible, actively and productively.

\section{Justification}

With this background as a premise and to meet the above objective it has been developed in Cuba for more than 20 years ago a cardiac rehabilitation program, implemented directly in the community (Primary Health Care level), sponsored by the Ministry of Public Health and coordinated by the National Group of Cardiology, which takes effect even today in all provinces of the country $[12,13]$.

For implementation of this program, the human and material resources available in each region of the country, is taken into consideration, and adaptations are made based on their socioeconomic conditions.

Population: The program included all those patients with any medical or surgical heart disease, congenital or acquired, with particular emphasis on cases with myocardial infarction or other form of ischemic heart disease [14].

\section{Activities}

\section{The program includes 3 phases:}

Phase I (Hospital): It takes place in all hospitals in the country where they admit patients with the diseases previously mentioned; they will involve professionals involved in the comprehensive care of these patients. During the exercise sessions, which will begin with passive and active physiotherapy, it will attempt to reach a conditioning pulse exceeding 20 beats $/ \mathrm{min}$ at baseline.

At the moment of the hospital discharge the patient will be sent to the family doctor or the corresponding polyclinic or primary health care center. A document stating the definitive diagnosis, complications and treatment, including the prescription of physical activity will also be sent.

Phase II (Convalescence): It will be carried out in the departments or rehabilitation centers of medical institutions, therapeutic areas and other selected places in the community or the primary health care system and will include specialists, family doctors, physiotherapists and other exercise specialists who develop the rehabilitation program. This phase will last approximately 8 weeks including supervised exercise sessions that will be held 3 to 5 times per week or daily or on alternate days, according to the possibilities of each place and will last for 30 to $45 \mathrm{~min}$. Training sessions include dynamic, rhythmic, repetitive physical exercises, with a necessary intensity so that the predetermined training pulse is reached. Calisthenics, walking, jogging, pedaling on a stationary bicycle, treadmill, paddles and other aerobic exercises can be included in the session (Figures 1 and 2).

Phase III (Maintenance): It starts with the return to work or social reintegration of the patient and will last indefinitely; will be held in the same places of Phase II but mainly in sports fields, gyms, parks and other places in the community with the minimum conditions required for the completion of the exercise program, for which it is not indispensable the use of sophisticated equipment or other resource materials. They involve mainly family doctors, physiatrists, physical therapists Physical Culture specialists, in supervising exercise sessions

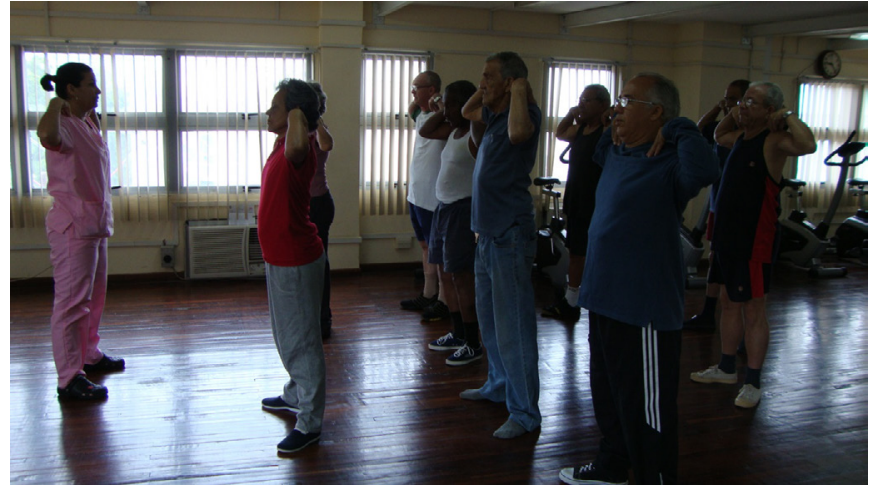

Figure 1: Patients with various types of cardiovascular diseases but mainly with different forms of ischemic heart disease while conducting a session of supervised exercise in a gym at the Rehabilitation Center of a hospital in the capital. Patients were clinically evaluated previously by a cardiologist; an individual training pulse was determined before.

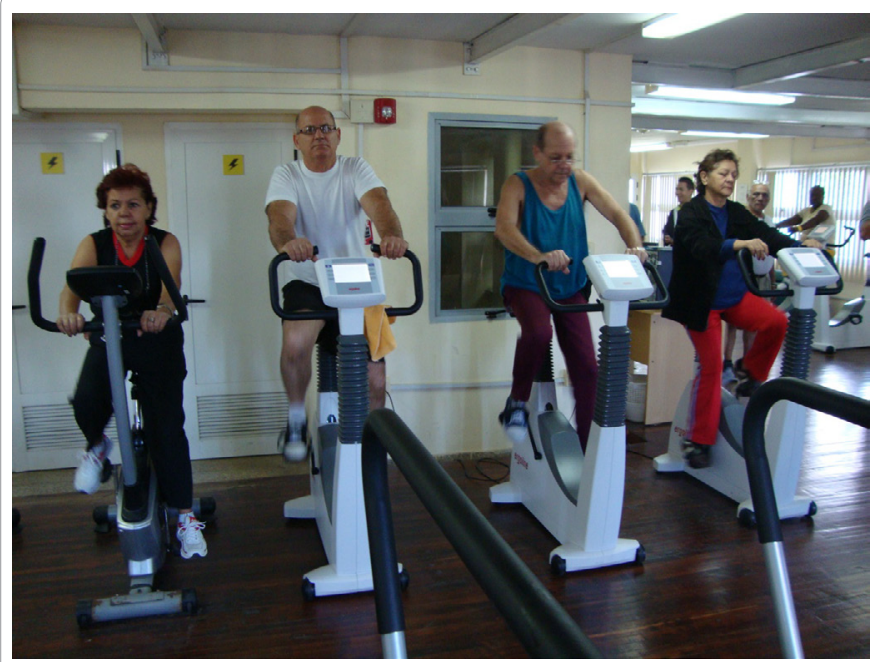

Figure 2: After calisthenics, made by way of "warming", patients perform a dynamic higher intensity exercise, in this case in a static training upright veloergometer, they can also be done on an endless belt. In all exercise sessions fundamental principles of physical training in cardiac patients are met.

and other professionals in the prescription and evaluation of the program.

The physical training program should follow the principles of the previous phase, trying the exercise be performed continuously at the determined intensity for a period of 15 to $30 \mathrm{~min}$; it must be made with a periodicity of not less than 3 times per week. This phase may be supplemented by the realization of collective sports games trying to achieve camaraderie between patients and greater adherence to the program [15].

The maintenance phase is indefinite, but it should be done with more emphasis in the first year after an acute episode, coronary angioplasty or surgery.

\section{Conclusion}

One of the main benefits expected from the implementation of this program is that a large majority of cardiac patients may be reinstated earlier and in better condition to their work or social activities. An improvement in functional capacity, symptom relief and reduction in 
Citation: Rivas-Estany E (2016) Physical Exercise in the Prevention and Rehabilitation of Cardiac Patients: The Cuban Experience in the Primary Health Care System. Primary Health Care 6: 243. doi:10.4172/2167-1079.1000243

consumption of prescribed drugs, decreased anxiety and depression should be observed. We will therefore see major preservation of the role of the patient in their family and social life, all of which result in significant socio-economic benefits for the country $[13,14]$.

\section{References}

1. Health Statistics Yearbook (2015) Republic of Cuba. Ministry of Public Health. Medical Records and Health Statistics Department. Havana, 2016.

2. Rivas Estany E, Ponce de León Aguilera O, Sin Chesa C, Gutiérrez Calderon F (1990) Comprehensive Cardiac Rehabilitation with early physical training in patients with myocardial infarction. Rev Cubana Cardiol Cir Cardiovasc 4: 177-189.

3. Lavie CJ, Thomas RJ, Squires RW, Allison TG, Milani RV (2009) Exercise training and Cardiac rehabilitation in primary and secondary prevention of coronary heart disease. Mayo Clin Proc 84: 373-383.

4. Archer E, Blair SN (2011) Physical activity and the prevention of cardiovascular disease: From evolution to epidemiology. Prog Cardiovasc Dis 53: 387-396.

5. Lavie CJ (2011) Exercise and cardiovascular diseases - a matter of life or death. Prog Cardiovasc Dis 53: 385-386.

6. Lavie CJ, Milani RV (2011) Cardiac rehabilitation and exercise training in secondary coronary heart disease prevention. Prog Cardiovasc Dis 53: 397-403.

7. Williams MA, Ades PA, Hamm LF, Keteyian SJ, LaFontaine TP, et al. (2006)
Clinical evidence for a health benefit from cardiac rehabilitation: An update. Am Heart J 152: 835-841.

8. Ades PA (2001) Cardiac rehabilitation and secondary prevention of coronary heart diseases. $\mathrm{N}$ Engl J Med 345: 892-902.

9. Wenger NK (2008) Current status of cardiac rehabilitation. J Am Coll Cardiol 51: 1619-1631.

10. American College of Sports Medicine: Guidelines for Exercise Testing and Prescriptions. 4th ed. Philadelphia: Lea \& Febiger; 1991.

11. Rivas Estany E (2011) Physical exercise in cardiovascular prevention and rehabilitation. Rev Esp Cardiol Supl 11: 18-22.

12. Project for a National Cardiac Rehabilitation Program in the Community (1989) Rev Cubana Cardiol Cir Cardiovasc 3: 244-259.

13. Rivas Estany E, Barrera Sarduy J, Sixto Fernández S, Rodríguez Nande LM, Kesser Garcia C (2013) Cuban cardiac rehabilitation program. Results. Rehabilitación (Madr.) 47: 238-244.

14. Rivas Estany E, Alvarez Gomez JA, Barrera Sarduy JD, Sixto Fernandez S, Rodriguez Nande LM, et al. (2008) Assessment of a national rehabilitation programme for patients after cardiovascular diseases in a developing country. Europ J Cardiovasc Prev Rehab, p: S50.

15. Rivas Estany E (2011) Prolonged cardiac rehabilitation. In: Maroto Montero JM de Pablo Zarzosa C (editors). Cardiovascular Rehabilitation, Madrid: Editorial Panamericana, pp: 463-472. 